Research Article

\title{
Elastic Analysis of Nonhomogeneous Frozen Wall under Nonaxisymmetric Ground Stress Field and in State of Unloading
}

\author{
Wen Zhang $\mathbb{D}^{D}$, Baosheng Wang $\mathbb{D}$, and Yong Wang \\ State Key Laboratory for Geomechanics and Deep Underground Engineering, China University of Mining and Technology, \\ Xuzhou 221116, China \\ Correspondence should be addressed to Baosheng Wang; wangbaosheng@cumt.edu.cn
}

Received 15 January 2018; Accepted 15 May 2018; Published 3 July 2018

Academic Editor: Aniello Riccio

Copyright (c) 2018 Wen Zhang et al. This is an open access article distributed under the Creative Commons Attribution License, which permits unrestricted use, distribution, and reproduction in any medium, provided the original work is properly cited.

The mechanical analysis of frozen walls is a cornerstone technology of artificially frozen ground. The mechanical response of frozen walls is affected by heterogeneity, excavation unloading, uneven ground pressure, and the characteristics of surrounding rock. However, these factors are rarely taken into full consideration in existing analysis models. To address this shortfall, this study presents a plane-strain model that considers the inhomogeneity of frozen walls, the unloaded state of the frozen-wall inner edge, and the nonuniform ground stress field (abbreviated as "IF model"). The solution of the IF model is based on the superposition of thin concentric cylinders under two types of contact conditions: complete contact and smooth contact, and its validity is tested by a finite-element calculation. The calculation indicates that the excavation reduces the radial force and increases the tangential force between the frozen wall and the surrounding earth mass; the ground principal stress is rotated after the excavation. If the radial unloading equals the tangential unloading at the inner edge of the frozen wall, the response of the radial stress differs from that of the tangential stress at the outer edge of the frozen wall. The circumferential stress and the radial displacement at the inner edge correlate linearly with the nonuniform coefficient of the ground press and the unloading ratio. If the nonuniform coefficient is relatively small, the inner edge of the frozen wall may incur tensile damage. Compared with the model of a homogeneous frozen wall (abbreviated as "HF model"), which has a uniform temperature distribution, the absolute value of the circumferential stress is lower (higher) for the IF model where the temperature is above (below) average. When the frozen wall is relatively thick, the circumferential stress of the inner edge of the frozen wall is lower for the IF model than for the HF model. The percent reduction is $8.12 \% \sim 9.32 \%$ for rock freezing and $13.41 \% \sim 18.03 \%$ for soil freezing. The IF model proposed herein thus reflects the characteristics of frozen walls and surrounding rock more clearly and accurately than the HF model and obtains stress states closer to the reality. Therefore, the IF model is recommended for the design and construction of frozen walls.

\section{Introduction}

Artificial ground freezing [1] is commonly used for excavation in water-bearing and unstable strata, such as shaft engineering $[2,3]$, tunnel construction [4-6], and foundation pit excavation [7]. The design of the frozen wall is one of critical technologies in this method, and the analysis of the mechanics is the foundation of this technology. Although the mechanical model of a frozen wall in a uniform ground stress field is fully developed [8-15], many underground structures, such as tunnels, subways, and inclined and vertical shafts in bedrock, are built in zones of uneven earth pressure [16-19]. To study such underground structures, three types of mechanical models have been developed: the loading model for homogeneous frozen walls $[3,20]$, the loading model for inhomogeneous frozen walls considering the radial variation of temperature, and the homogeneous unloading model considering excavation unloading and the interaction between the homogeneous frozen wall and surrounding rock [21, 22]. However, these three models still do not accurately reflect the characteristics of an inhomogeneous frozen wall during unloading. To address this challenge, the present study proposes a plane-strain model that considers the inhomogeneity of the frozen wall and excavation unloading under a nonuniform ground stress field. The model is based on the radial superposition of thin 


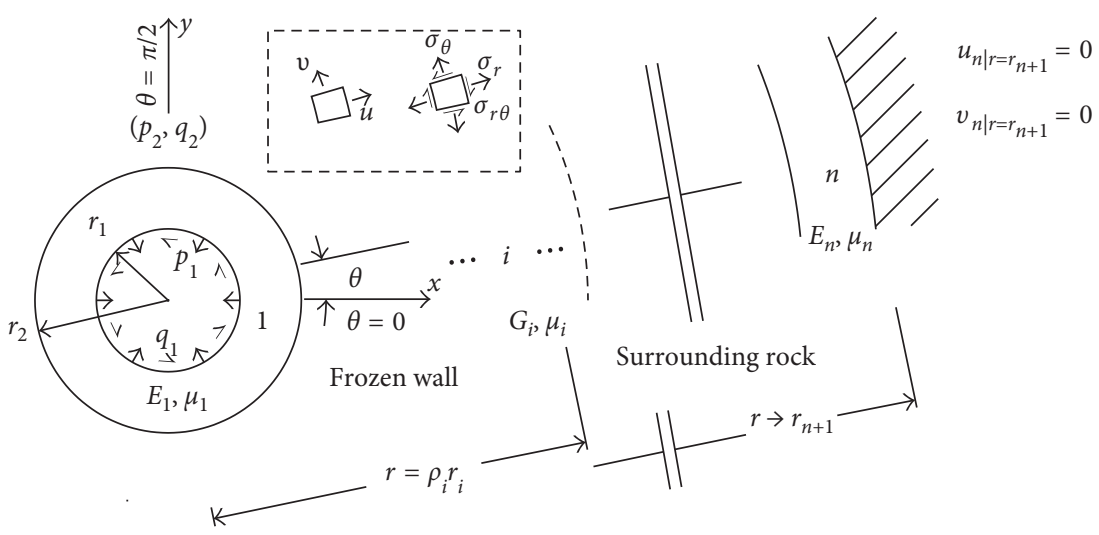

Figure 1: Mechanical model I: the mechanics model for interactions between a heterogeneous frozen wall and surrounding rock in the unloading state.

cylinders (Figure 1), and its analytic solution is derived and verified against results from a finite-element simulation. Next, the results of the proposed model are compared with those of the homogeneous model, and conclusions are drawn. Throughout this paper, the positive and negative signs of stress and displacement comply with the regularity of elastic mechanics. The subscripts $r, \theta$, and $r \theta$ indicate the radial, circumferential, and shear components, respectively. The subscript " $i$ " indicates the layer number in the cylinder.

\section{Mechanical Model}

2.1. Nondimensionalization of Parameters. To simplify the analysis, we select appropriate normalized variables to transform the parameters into dimensionless form. The regular variable with units is indicted by adding a caret $(\wedge)$ above it. The inner radius $\widehat{r}_{i}$ of each layer is used to scale displacement. $u=\widehat{u} / \widehat{r}_{i}$ and $v=\widehat{v} / \widehat{r}_{i}$ are the dimensionless radial displacement and circumferential displacement, respectively. $\rho_{i}=\widehat{r} / \widehat{r}_{i} \quad\left(\widehat{r}_{i} \leq \widehat{r} \leq \widehat{r}_{i+1}\right)$ is the nondimensional radial coordinate of the $i$ th cylinder from the cylinder axis. $r_{i}$ and $r_{i+1}$ are the inner and outer radii of the $i$ th cylinder, respectively. $r=\widehat{r} / \widehat{r}_{1}$ indicates the radial coordinate spanning over the entire coordinate system, and $\widehat{r}_{1}$ is the inner radius of the frozen wall. Also, the maximum principal ground pressure $\widehat{p}_{y}$ is used to scale the relevant stress and pressure. $p=\widehat{p} / \widehat{p}_{y}$ and $q=\widehat{q} / \widehat{p}_{y}$ are the dimensionless radial load and tangential load on the cylinder surface, respectively. $\sigma_{r}=\widehat{\sigma}_{r} / \widehat{p}_{y}, \sigma_{\theta}=\widehat{\sigma}_{\theta} / \widehat{p}_{y}, \sigma_{r \theta}=\widehat{\sigma}_{r \theta} / \widehat{p}_{y}, E=\widehat{E} / \widehat{p}_{y}$, and $G=\widehat{G} / \widehat{p}_{y}$ are the dimensionless radial stress, hoop stress, shear stress, Young's modulus, and shear modulus, respectively. Note that the initial horizontal principal stress is $\widehat{\sigma}_{x}^{0}=\widehat{p}_{x}=\lambda \widehat{p}_{y}$ and the initial vertical principal stress is $\hat{\sigma}_{y}^{0}=\widehat{p}_{y}$. Therefore, the initial dimensionless ground principal stresses are $\sigma_{x}^{0}=\lambda$ and $\sigma_{y}^{0}=1$, where $\lambda\left(0 \leq \lambda=\widehat{p}_{x} / \widehat{p}_{y} \leq 1\right)$ is the nonuniform coefficient of ground press.

2.2. Fundamental Assumptions. We make the following fundamental assumptions:

(1) Both the frozen wall and surrounding rock consist of linear elastic material under plane-strain conditions, and their physical and mechanical parameters depend on the radius.

(2) The initial geostress field is fixed before and after freezing, and the initial principal stresses are $\sigma_{x}^{0}=\lambda$ and $\sigma_{y}^{0}=1$. Switching to polar coordinates gives

$$
\begin{aligned}
\sigma_{r}^{0} & =-\frac{1+\lambda}{2}+\frac{1-\lambda}{2} \cos 2 \theta, \\
\sigma_{\theta}^{0} & =-\frac{1+\lambda}{2}-\frac{1-\lambda}{2} \cos 2 \theta, \\
\sigma_{r \theta}^{0} & =-\frac{1-\lambda}{2} \sin 2 \theta,
\end{aligned}
$$

where $\theta$ is the angular coordinate (Figure 1). $\theta=0$ and $\theta=$ $\pi / 2$ give the directions of minimum and maximum crustal stress ( $x$ and $y$ directions), respectively.

2.3. Boundary Conditions. The analytical solution of the problem is the superposition of solutions for the initial stratum stress or displacement and the solutions of the mechanical model I (Figure 1). The frozen wall and surrounding rock are divided into $n$ concentric layered cylinders, which are regarded as homogeneous, isotropic, and linear elastic. These cylinders remain in the range of small deformations. The number $n$ of cylinder layers depends on the radial temperature profile $T(r)$. For model $\mathrm{I}$, the loads on the inner $\left(r=r_{i}\right)$ and outer $\left(r=r_{i+1}\right)$ surfaces are

$$
\begin{aligned}
p_{i} & =\delta_{i}+\xi_{i} \cos 2 \theta, \\
q_{i} & =\omega_{i} \sin 2 \theta, \\
p_{i+1} & =\delta_{i+1}+\xi_{i+1} \cos 2 \theta, \\
q_{i+1} & =\omega_{i+1} \sin 2 \theta .
\end{aligned}
$$

The displacement condition on the external boundary of surrounding rock $\left(r=r_{n+1}\right)$ is

$$
\begin{aligned}
& u_{n \mid r=r_{n+1}}=0, \\
& v_{n \mid r=r_{n+1}}=0 .
\end{aligned}
$$




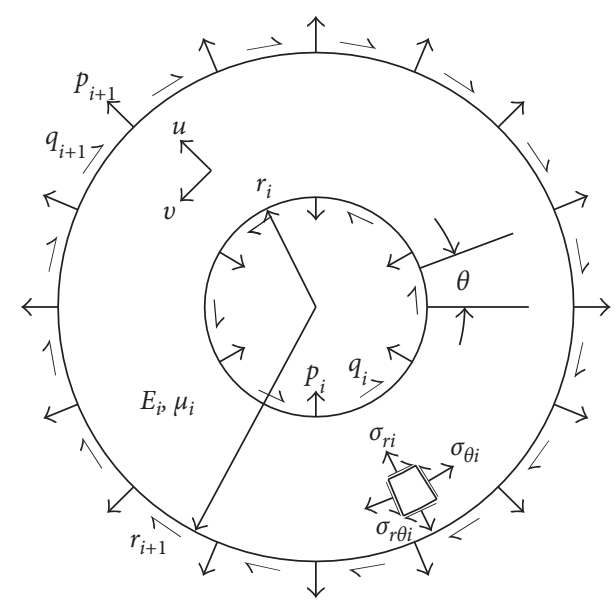

FIgURE 2: Mechanical model II: single-layer model.

The stress boundary condition on the inner edge of the frozen wall $\left(r=r_{1}=1\right)$ is

$$
\begin{aligned}
& p_{1}=-\eta \sigma_{r}^{0}, \\
& q_{1}=-\eta \sigma_{r \theta}^{0},
\end{aligned}
$$

where $\eta$ is the unloading ratio of the internal surface of the frozen wall caused by the excavation $(0 \leq \eta \leq 1, \eta=1$ when completely unloaded and $\eta=0$ when not excavated). The value of the "unloading ratio" is determined by the construction scheme, the lining structure, and the mechanical properties of surrounding ground (such as stiffness and Poisson's ratio) [23].

\section{Solution of Model}

3.1. Basic Solution. The mechanical model I can be decomposed into many single-layer models, which we call model II (Figure 2).

The analytical solution $[17,18]$ of model II is

$$
\begin{gathered}
\sigma_{r i}=h_{r i}\left(\rho_{i}\right)+g_{r i}\left(\rho_{i}\right) \cdot \cos 2 \theta, \\
h_{r i}\left(\rho_{i}\right)=2 a_{i}+b_{i} \rho_{i}^{-2}, \\
g_{r i}\left(\rho_{i}\right)=-2 f_{i}-4 c_{i} \rho_{i}^{-2}-6 e_{i} \rho_{i}^{-4}, \\
\sigma_{\theta i}=h_{\theta i}\left(\rho_{i}\right)+g_{\theta i}\left(\rho_{i}\right) \cdot \cos 2 \theta, \\
h_{\theta i}\left(\rho_{i}\right)=2 a_{i}-b_{i} \rho_{i}^{-2}, \\
g_{\theta i}\left(\rho_{i}\right)=2 f_{i}+12 d_{i} \rho_{i}^{2}+6 e_{i} \rho_{i}^{-4}, \\
\sigma_{r \theta i}=h_{r \theta i}\left(\rho_{i}\right) \cdot \sin 2 \theta, \\
h_{r \theta i}\left(\rho_{i}\right)=6 d_{i} \rho_{i}^{2}+2 f_{i}-2 c_{i} \rho_{i}^{-2}-6 e_{i} \rho_{i}^{-4}, \\
u_{i}=h_{u i}\left(\rho_{i}\right)+g_{u i}\left(\rho_{i}\right) \cdot \cos 2 \theta, \\
h_{u i}\left(\rho_{i}\right)=-G_{i}^{-1}\left(a_{i} \rho_{i} \alpha_{i}+0.5 b_{i} \rho_{i}^{-1}\right), \\
g_{u i}\left(\rho_{i}\right)=G_{i}^{-1}\left(e_{i} \rho_{i}^{-3}-2 d_{i} \mu_{i} \rho_{i}^{3}-f_{i} \rho_{i}-2 c_{i} \rho_{i}^{-1} \beta_{i}\right), \\
v_{i}=h_{v i}\left(\rho_{i}\right) \cdot \sin 2 \theta \\
h_{v i}\left(\rho_{i}\right)=G_{i}^{-1}\left(f_{i} \rho_{i}+c_{i} \rho_{i}^{-1} \alpha_{i}+e_{i} \rho_{i}^{-3}-d_{i} \rho_{i}^{3} \gamma_{i}\right),
\end{gathered}
$$

where $\quad \alpha_{i}=2 \mu_{i}-1, \quad \beta_{i}=\mu_{i}-1, \quad \gamma_{i}=2 \mu_{i}-3, \quad \rho_{i} \in\left[1, k_{i}\right]$, $k_{i}=\widehat{r}_{i+1} / \hat{r}_{i}, G_{i}=0.5 E_{i} /\left(1+\mu_{i}\right)$, and $\mu_{i}$ is Poisson's ratio. The coefficients $a_{i}$ to $f_{i}$ are related to the boundary conditions. When $i=1,2, \ldots, n-1$, the values of $a_{i}$ to $f_{i}$ are as follows:

$$
\begin{aligned}
a_{i} & =-A_{i}^{-1}\left(\delta_{i}-\delta_{i+1} k_{i}^{2}\right) / 2, \\
b_{i} & =A_{i}^{-1} k_{i}^{2}\left(\delta_{i}-\delta_{i+1}\right), \\
c_{i} & =A_{i}^{-3} k_{i}^{2}\left(k_{i}^{2} B_{i} \omega_{i}-C_{i} \xi_{i}+D_{i} \xi_{i+1}-B_{i} \omega_{i+1}\right) / 2, \\
d_{i} & =A_{i}^{-3}\left(F_{i} \omega_{i}-H_{i} \xi_{i}+k_{i}^{2} I_{i} \xi_{i+1}+k_{i}^{2} K_{i} \omega_{i+1}\right) / 6, \\
e_{i} & =-A_{i}^{-3} k_{i}^{4}\left(2 k_{i}^{2} \omega_{i}-I_{i} \xi_{i}+H_{i} \xi_{i+1}-2 \omega_{i+1}\right) / 6, \\
f_{i} & =-A_{i}^{-3}\left(2 k_{i}^{4} \omega_{i}-D_{i} \xi_{i}+k_{i}^{2} C_{i} \xi_{i+1}-2 k_{i}^{2} \omega_{i+1}\right) / 2, \\
A_{i} & =k_{i}^{2}-1, \\
B_{i} & =k_{i}^{2}+1, \\
C_{i} & =k_{i}^{4}+k_{i}^{2}+2, \\
D_{i} & =2 k_{i}^{4}+k_{i}^{2}+1, \\
F_{i} & =3 k_{i}^{2}-1, \\
H_{i} & =3 k_{i}^{2}+1, \\
I_{i} & =k_{i}^{2}+3, \\
K_{i} & =k_{i}^{2}-3 .
\end{aligned}
$$

When $i=n$, we have $\sigma_{r n \mid r=r_{n}}=p_{n}$ and $\sigma_{r \theta n \mid r=r_{n}}=q_{n}$. The coefficients $a_{n}$ to $f_{n}$ can be obtained by using (3). The result is

$$
\begin{aligned}
& a_{n}=-0.5 A_{n}^{-1} \delta_{n}, \\
& b_{n}=A_{n}^{-1} \delta_{n}\left(A_{n}+1\right), \\
& c_{n}=-B_{n}^{-1} k_{n}^{2}\left(C_{n} \omega_{n}+D_{n} \xi_{n}\right), \\
& d_{n}=-B_{n}^{-1}\left(F_{n} \omega_{n}+H_{n} \xi_{n}\right), \\
& e_{n}=-B_{n}^{-1} k_{n}^{4}\left(I_{n} \omega_{n}+J_{n} \xi_{n}\right), \\
& f_{n}=B_{n}^{-1}\left(K_{n} \omega_{n}+L_{n} \xi_{n}\right), \\
& A_{n}=\alpha_{n} k_{n}^{2}-1, \\
& B_{n}=6\left[\left(3-4 \mu_{n}\right)\left(k_{n}^{8}+1\right)+4 k_{n}^{6}\left(4 \mu_{n}^{2}-6 \mu_{n}+3\right)-2 k_{n}^{2}\left(3 k_{n}^{2}-2\right)\right], \\
& C_{n}=3 k_{n}^{2}\left(4 k_{n}^{4} \mu_{n}-3 k_{n}^{4}-1\right), \\
& D_{n}=-12 k_{n}^{6} \mu_{n}+9 k_{n}^{6}-3 k_{n}^{2}+6, \\
& F_{n}=3 k_{n}^{4}-4 k_{n}^{2}+4 \mu_{n}-3, \\
& H_{n}=-3 k_{n}^{4}+2 k_{n}^{2}+4 \mu_{n}-3, \\
& I_{n}=-2 k_{n}^{2}\left(4 k_{n}^{2} \mu_{n}-3 k_{n}^{2}-4 \mu_{n}^{2}+6 \mu_{n}-3\right), \\
& J_{n}=k_{n}^{4}\left(4 \mu_{n}-3\right)+2 k_{n}^{2}\left(4 \mu_{n}^{2}-6 \mu_{n}+3\right)-3, \\
& K_{n}=6 k_{n}^{4}\left(4 k_{n}^{2} \mu_{n}^{2}-6 k_{n}^{2} \mu_{n}+3 k_{n}^{2}-1\right), \\
& L_{n}=-6 k_{n}^{6}\left(4 \mu_{n}^{2}-6 \mu_{n}+3\right)+3 k_{n}^{4}+12 \mu_{n}-9 .
\end{aligned}
$$


When $k_{n} \rightarrow \infty$, we obtain

$$
\begin{aligned}
& a_{n}=0, \\
& b_{n}=\delta_{n}, \\
& c_{n}=0.5\left(\omega_{n}-\xi_{n}\right), \\
& d_{n}=0, \\
& e_{n}=\left(\xi_{n}-2 \omega_{n}\right) / 6, \\
& f_{n}=0 .
\end{aligned}
$$

3.2. Solution for Mechanical Model I. The contact condition between each single layer in mechanical model I involves two different limited states: complete contact and smooth contact [17, 24-27]. Obviously, in the analysis of the heterogeneous frozen wall, the contact condition is complete contact. In addition, to make the solution of model I more universal and applicable to more engineering problems, we also solve model I for the condition of smooth contact.

3.2.1. Complete Contact. For complete contact, the radial stresses at the interfaces between contact thin cylinders are equal and displacements at the contact interface are continuous. Thus, according to the compatibility condition for displacements, we obtain

$$
\begin{aligned}
& u_{j \mid r=r_{j+1}} \widehat{r}_{j}=u_{j+1 \mid r=r_{j+1}} \widehat{r}_{j+1}, \\
& v_{j \mid r=r_{j+1}} \widehat{r}_{j}=\left.v_{j+1}\right|_{r=r_{j+1}} \widehat{r}_{j+1},
\end{aligned}
$$

where $j=1,2, \ldots, n-1$ is the contact-surface number (from interior to exterior). The above boundary conditions are applicable for arbitrary $\theta$, so we get solutions simultaneously with (5)-(9):

$$
\begin{aligned}
& h_{u j}\left(r_{j+1}\right) \widehat{r}_{j}=h_{u(j+1)}\left(r_{j+1}\right) \widehat{r}_{j+1}, \\
& g_{u j}\left(r_{j+1}\right) \widehat{r}_{j}=g_{u(j+1)}\left(r_{j+1}\right) \widehat{r}_{j+1}, \\
& h_{v j}\left(r_{j+1}\right) \widehat{r}_{j}=h_{v(j+1)}\left(r_{j+1}\right) \widehat{r}_{j+1} .
\end{aligned}
$$

Arranging (14)-(16), we get

$$
\begin{gathered}
M_{j} \delta_{j}+N_{j} \delta_{j+1}+O_{j} \delta_{j+2}=0, \\
P_{j} \xi_{j}+Q_{j} \xi_{j+1}+R_{j} \xi_{j+2}+S_{j} \omega_{j} \\
+\chi_{j} \cdot(s+t) \cdot T_{j} \omega_{j+1}+U_{j} \omega_{j+2}=0, \\
\chi_{j} \cdot(s+t) \cdot\left(V_{j} \xi_{j}+W_{j} \xi_{j+1}+X_{j} \xi_{j+2}\right. \\
\left.+Y_{j} \omega_{j}+Z_{j} \omega_{j+1}+\Pi_{j} \omega_{j+2}\right)=0,
\end{gathered}
$$

where $\chi_{j}$ is the state parameter of the interface and $\chi_{j}=1$ when contact is complete. When $j=1,2, \ldots, n-2, s=1$ and $t=0$; when $j=n-1, s=0$ and $t=1$ :

$$
\begin{aligned}
& \varsigma_{j}=A_{j+1} / A_{j} \\
& M_{j}=\varsigma_{j}\left(\alpha_{j}-1\right) \text {, } \\
& m_{j}=G_{j} / G_{j+1} \text {, } \\
& N_{j}=\varsigma_{j}\left(1-k_{j}^{2} \alpha_{j}\right)-m_{j}\left[s\left(\alpha_{j+1}-k_{j+1}^{2}\right)-t\left(\alpha_{j+1}-A_{j+1}+1\right)\right] \text {, } \\
& O_{j}=-s \cdot m_{j}\left(1-\alpha_{j+1}\right) k_{j+1}^{2} \text {, } \\
& P_{j}=\varsigma_{j}^{3}\left(2 H_{j} \mu_{j} k_{j}^{2}-6 D_{j}+6 C_{j} \beta_{j}+I_{j}\right) \text {, } \\
& Q_{j}=\varsigma_{j}^{3}\left(6 k_{j}^{2} C_{j}-2 I_{j} k_{j}^{4} \mu_{j}-6 D_{j} \beta_{j}-H_{j}\right) \\
& +m_{j}\left[s\left(6 D_{j+1}-2 H_{j+1} \mu_{j+1}-6 k_{j+1}^{2} C_{j+1} \beta_{j+1}-k_{j+1}^{4} I_{j+1}\right)\right. \\
& +t 6 A_{j+1}^{3} B_{j+1}^{-1}\left(L_{j+1}-2 H_{j+1} \mu_{j+1}\right. \\
& \left.\left.-2 k_{j+1}^{2} D_{j+1} \beta_{j+1}+k_{j+1}^{4} J_{j+1}\right)\right] \text {, } \\
& R_{j}=s m_{j} k_{j+1}^{2}\left(2 I_{j+1} \mu_{j+1}-6 C_{j+1}+6 D_{j+1} \beta_{j+1}-k_{j+1}^{2} H_{j+1}\right) \text {, } \\
& S_{j}=2 \varsigma_{j}^{3} k_{j}^{2}\left(6 k_{j}^{2}-F_{j} \mu_{j}-3 B_{j} \beta_{j}-1\right), \\
& T_{j}=2 \varsigma_{j}^{3}\left(3 B_{j} \beta_{j}-k_{j}^{4} K_{j} \mu_{j}-6 k_{j}^{2}+1\right) \\
& +m_{j}\left[2 s\left(F_{j+1} \mu_{j+1}-6 k_{j+1}^{4}+3 k_{j+1}^{4} B_{j+1} \beta_{j+1}+k_{j+1}^{6}\right)\right. \\
& +t 6 A_{j+1}^{3} B_{j+1}^{-1}\left(K_{j+1}-2 F_{j+1} \mu_{j+1}\right. \\
& \left.\left.-2 k_{j+1}^{2} C_{j+1} \beta_{j+1}+k_{j+1}^{4} I_{j+1}\right)\right] \text {, } \\
& U_{j}=s \cdot 2 m_{j} k_{j+1}^{2}\left(K_{j+1} \mu_{j+1}+6-3 B_{j+1} \beta_{j+1}-k_{j+1}^{2}\right) \text {, } \\
& V_{j}=\varsigma_{j}^{3}\left(6 D_{j}-3 C_{j} \alpha_{j}+I_{j}+H_{j} k_{j}^{2} \gamma_{j}\right) \text {, } \\
& W_{j}=\varsigma_{j}^{3}\left(3 D_{j} \alpha_{j}-6 k_{j}^{2} C_{j}-H_{j}-I_{j} k_{j}^{4} \gamma_{j}\right) \\
& +m_{j}\left[s\left(3 k_{j+1}^{2} C_{j+1} \alpha_{j+1}-6 D_{j+1}-k_{j+1}^{4} I_{j+1}-H_{j+1} \gamma_{j+1}\right)\right. \\
& +6 t A_{j+1}^{3} B_{j+1}^{-1}\left(k_{j+1}^{2} D_{j+1} \alpha_{j+1}-L_{j+1}\right. \\
& \left.\left.+k_{j+1}^{4} J_{j+1}-H_{j+1} \gamma_{j+1}\right)\right] \text {, } \\
& X_{j}=m_{j} k_{j+1}^{2}\left(6 C_{j+1}-3 D_{j+1} \alpha_{j+1}+k_{j+1}^{2} H_{j+1}+I_{j+1} \gamma_{j+1}\right) \text {, } \\
& Y_{j}=\varsigma_{j}^{3} k_{j}^{2}\left(3 B_{j} \alpha_{j}-12 k_{j}^{2}-2-F_{j} \gamma_{j}\right) \text {, } \\
& Z_{j}=\varsigma_{j}^{3}\left(12 k_{j}^{2}-3 B_{j} \alpha_{j}+2-k_{j}^{4} K_{j} \gamma_{j}\right) \\
& +m_{j}\left[s\left(12 k_{j+1}^{4}-3 k_{j+1}^{4} B_{j+1} \alpha_{j+1}+2 k_{j+1}^{6}+F_{j+1} \gamma_{j+1}\right)\right. \\
& +6 t A_{j+1}^{3} B_{j+1}^{-1}\left(k_{j+1}^{2} C_{j+1} \alpha_{j+1}-K_{j+1}\right. \\
& \left.\left.+k_{j+1}^{4} I_{j+1}-F_{j+1} \gamma_{j+1}\right)\right] \text {, } \\
& \Pi_{j}=s \cdot m_{j} k_{j+1}^{2}\left(3 B_{j+1} \alpha_{j+1}-12-2 k_{j+1}^{2}+K_{j+1} \gamma_{j+1}\right) \text {. }
\end{aligned}
$$

3.2.2. Smooth Contact. For smooth contact, normal stresses and radial displacements at the contact interface are continuous and the tangential stress is zero $\left(q_{j+1}=0\right)$. We can 


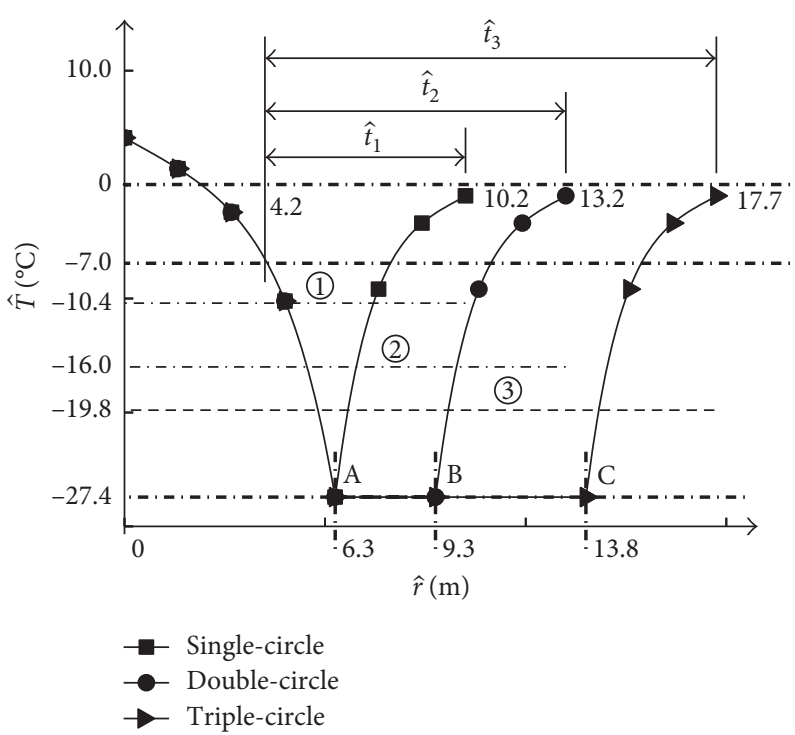

FIgURE 3: Temperature $\widehat{T}$ as a function of radius for different freezing modes.

still use (17)-(19), but the state parameter of the interface is zero $\left(\chi_{j}=0\right)$. Also, $\omega_{j+1}=0$ under these conditions.

3.2.3. Solving Equations of Model I. There are $n-1$ contact interfaces in an $n$-layered cylinder. Substituting $j=1 \sim n-1$ into (17)-(19), we obtain a $3(n-1)$-degree linear equation with $\delta_{2}, \xi_{2}, \omega_{2}, \ldots, \delta_{n}, \xi_{n}, \omega_{n}$ as variables. This can be solved directly by using mathematical calculation software. Next, substituting the result into (5)-(9) gives the analytical solutions for stress and displacement of each layer in model I.

3.3. Stress and Displacement Solutions for Frozen Wall and Surrounding Rock. The stress and displacement solutions for the frozen wall and surrounding rock are the sum of the solutions in Section 3.2 and the initial stress field and displacement field (initial displacement field is zero). The total solution is expressed by adding an asterisk $\left({ }^{*}\right)$ above the variable:

$$
\begin{aligned}
\sigma_{r i}^{*} & =\sigma_{r i}+\sigma_{r}^{0}, \\
\sigma_{\theta i}^{*} & =\sigma_{\theta i}+\sigma_{\theta}^{0}, \\
\sigma_{r \theta i}^{*} & =\sigma_{r \theta i}+\sigma_{r \theta}^{0}, \\
u_{i}^{*} & =u_{i}, \\
v_{i}^{*} & =v_{i} .
\end{aligned}
$$

\section{Comparison with Homogeneous Unloading Model}

The homogeneous unloading model (abbreviated as "HF model") regards the frozen wall and surrounding rock as homogeneous materials and thus uses average temperatures instead of considering radial variations in temperature. We compare the HF model with the inhomogeneous model (abbreviated as "IF model") and analyze the stress and displacement given by the two models under three freezing modes (single-circle pipes, double-circle pipes, and triplecircle pipes) and two formation conditions (rock freezing and soil freezing). For rock freezing, the elastic modulus varies only slightly upon freezing. However, the elastic modulus of soil increases significantly upon freezing.

4.1. Calculation Parameters. Figure 3 shows the distribution of temperature $\widehat{T}$ for different freezing modes. The thicknesses of the frozen wall for single-circle, double-circle, and triple-circle freezing are $\widehat{t}_{1}, \widehat{t}_{2}$, and $\widehat{t}_{3}$, respectively. Horizontal lines (1), (2), and (3) give the average temperatures for each of these three freezing modes. Points $A, B$, and $C$ show the location of the inner-, middle-, and outer-ring freezing pipes.

The basic parameters in the comparison include the depth $(150 \mathrm{~m})$, excavating radius $\left(\widehat{r}_{1}=4.2 \mathrm{~m}\right)$, exterior boundary $(100 \mathrm{~m})$, soil specific weight $\left(0.02 \mathrm{MN} / \mathrm{m}^{3}\right)$, unloading rate $(\eta=0.7)$, and nonuniform coefficient of ground press $(\lambda=0.65)$. The frozen wall is divided into several coaxial cylinders of identical thickness $(0.1 \mathrm{~m})$. The temperature of each cylinder is obtained from linear interpolation in Figure 3. The nonfrozen stratum is treated as a single outer cylinder, and the temperature of this region is assumed to be $25^{\circ} \mathrm{C}$.

For the IF model, the elastic modulus $\widehat{E}$ and Poisson's ratio $\mu$ in frozen rock can be expressed as

$$
\begin{aligned}
\widehat{E} & =-264.15 \cdot \widehat{T}+15604, \\
\mu & =0.0012 \cdot \widehat{T}+0.19 .
\end{aligned}
$$

In frozen soil, they are

$$
\begin{aligned}
\widehat{E} & =-22.453 \cdot \widehat{T}+721.32, \\
\mu & =0.0018 \cdot \widehat{T}+0.295 .
\end{aligned}
$$


TABLE 1: Mechanical parameters of frozen wall and surrounding earth mass for the HF model.

\begin{tabular}{|c|c|c|c|c|c|}
\hline Freezing modes & Average temperature $\left({ }^{\circ} \mathrm{C}\right)$ & Formation conditions & Mechanical parameters & Frozen wall & Surrounding earth mass \\
\hline \multirow{4}{*}{ Single-circle pipes } & \multirow{4}{*}{-10.4} & \multirow{2}{*}{ (1) rock freezing } & $\widehat{E}(\mathrm{MPa})$ & 18351 & 9000 \\
\hline & & & $\mu$ & 0.178 & 0.22 \\
\hline & & \multirow{2}{*}{ (2) soil freezing } & $\widehat{E}(\mathrm{MPa})$ & 955 & 160 \\
\hline & & & $\mu$ & 0.276 & 0.34 \\
\hline \multirow{4}{*}{ Double-circle pipes } & \multirow{4}{*}{-16} & \multirow{2}{*}{ (1) rock freezing } & $\widehat{E}(\mathrm{MPa})$ & 19830 & 9000 \\
\hline & & & $\mu$ & 0.171 & 0.22 \\
\hline & & \multirow{2}{*}{ (2) soil freezing } & $\widehat{E}(\mathrm{MPa})$ & 1081 & 160 \\
\hline & & & $\mu$ & 0.266 & 0.34 \\
\hline \multirow{4}{*}{ Triple-circle pipes } & \multirow{4}{*}{-19.8} & \multirow{2}{*}{ (1) rock freezing } & $\widehat{E}(\mathrm{MPa})$ & 20834 & 9000 \\
\hline & & & $\mu$ & 0.166 & 0.22 \\
\hline & & \multirow{2}{*}{ (2) soil freezing } & $\widehat{E}(\mathrm{MPa})$ & 1166 & 160 \\
\hline & & & $\mu$ & 0.259 & 0.34 \\
\hline
\end{tabular}

For the HF model, Table 1 gives the elastic modulus $\widehat{E}$ and Poisson's ratio $\mu$ corresponding to the average temperatures.

4.2. Verification of Analytical Solutions. To verify the analytical solution, we compare its predictions with the results of a finite-element model of a quarter of an ice wall $(0-\pi / 2)$ for single-cycle pipe freezing (Figure 4).

Figure 5 shows the distribution of radial stress $\sigma_{r}^{*}$, tangential stress $\sigma_{\theta}^{*}$, and radial displacement $u^{*}$ along the inner and outer edges of the frozen wall. The scatter plot shows the results of the finite-element calculation, and the curve shows the analytical results. The result of the analytical solution is consistent with the result of the finite-element calculation, which verifies the analytical solution.

4.3. Relationships between Stress and Displacement of Inhomogeneous Frozen Wall and $\lambda$ and $\eta$. For single-cycle pipe freezing, Figures 6(a) and 6(b) show the tangential stress $\sigma_{\theta}^{*}$ and radial displacement $u^{*}$, respectively, along the inner edge of the inhomogeneous frozen wall as a function of the nonuniform coefficient of ground press $\lambda$ and of the excavation unloading ratio $\eta . \sigma_{\theta}^{*}$ and $u^{*}$ are linearly correlated with $\lambda$ and $\eta$, which is consistent with the accumulative theory of linear elasticity. Thus, we deduce that the stress and displacement of any point in the frozen wall are also linear in $\lambda$ and $\eta$.

According to the elastic stress solution for a thick-walled cylinder, the weakest point is at the inner edge under the limit state, so we focus on the magnitudes of $\sigma_{\theta}^{*}$ and $u^{*}$ at the inner edge of the inhomogeneous frozen wall. For the orientation $\theta=0$, Figure 6 (a) shows the circumferential compressive stress concentration, and the inner edge of the frozen wall expands outward ( $u^{*}$ is positive) when $\lambda$ is relatively small $(\lambda \leq 0.43)$. As $\lambda$ increases, the circumferential compressive stress gradually decreases and the inner edge of the frozen wall begins to move inward instead of expanding. However, the dependencies are opposite when $\theta=\pi / 2$. The inner edge of the frozen wall incurs tensile stress with small $\lambda$, which degrades the safety of the frozen wall. To solve this problem, we can add auxiliary freezing holes in the direction $\theta=\pi / 2$ or adopt the freezing model of oval-ring pipes to avoid tensile damage at the inner edge of the frozen wall.

Figure 6(b) shows the tangential stress $\sigma_{\theta}^{*}$ and radial displacement $u^{*}$ of the inner edge as a function of the excavation unloading ratio $\eta$. As $\eta$ increases, the circumferential compressive stress concentrates significantly at $\theta=0$, and the inner edge shrinks much more in the direction $\theta=\pi / 2$.

4.4. Stress Variation at Outer Edge of Frozen Wall due to Excavation Unloading. Initially, we solve for the stress variation. For a homogeneous frozen wall and surrounding rock, we have $n=2$ and $j=1$ in (17)-(19). Thus, the interface mechanical parameters $\delta_{2}, \xi_{2}$, and $\omega_{2}$ can be obtained and then substituted into (21) to get the interaction force on the interface:

$$
\begin{aligned}
& p_{2}=\sigma_{r 2}^{*}=-\frac{1+\lambda}{2}\left(1-\phi_{1} \eta\right)+\frac{1-\lambda}{2}\left(1-\phi_{2} \eta\right) \cos 2 \theta \\
& q_{2}=\sigma_{r \theta 2}^{*}=-\frac{1-\lambda}{2}\left(1-\phi_{3} \eta\right) \sin 2 \theta
\end{aligned}
$$

where

$$
\begin{aligned}
& \phi_{1}=-M_{1} / N_{1}, \\
& \phi_{2}=\left[\left(2 A_{1} k_{1}^{4}+5 A_{1}+4\right) \omega_{2}-2 k_{1}^{2}\left(k_{1}^{2}+\omega_{1}\right)-D_{1}\right] / A_{1}^{3}, \\
& \phi_{3}=-\left[D_{1}-k_{1}^{2} C_{1} \omega_{2}+2 k_{1}^{4}+\left(K_{1} k_{1}^{4}+5 A_{1}+4\right) \omega_{1}\right] / A_{1}^{3}, \\
& \omega_{1}=\left[P_{1} W_{1}-Q_{1} V_{1}+Q_{1} Y_{1}-S_{1} W_{1}\right] /\left(Q_{1} Z_{1}-T_{1} W_{1}\right), \text { and } \\
& \omega_{2}=-\left(P_{1} Z_{1}-S_{1} Z_{1}-T_{1} V_{1}+T_{1} Y_{1}\right) /\left(Q_{1} Z_{1}-T_{1} W_{1}\right) .
\end{aligned}
$$

Comparing (24) with (1), we see that the unloading rates differ for the radial and tangential pressure.

(1) For the radial pressure $p_{2}$, the unloading proportions of the uniform term and nonuniform term are $\phi_{1} \eta$ and $\phi_{2} \eta$, respectively.

(2) The unloading rate for the tangential pressure $q_{2} \neq p_{2}$ is $\phi_{3} \eta$.

After numerous calculations, we find that the interface pressure of a radially inhomogeneous frozen wall can also be expressed by (24), except that the parameters $\phi_{1}, \phi_{2}$, and $\phi_{3}$ 


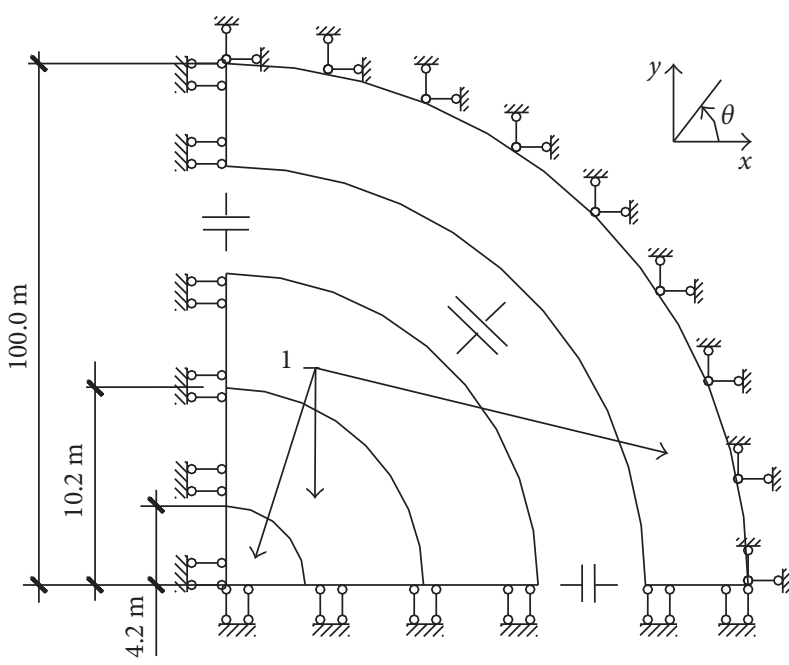

(a)

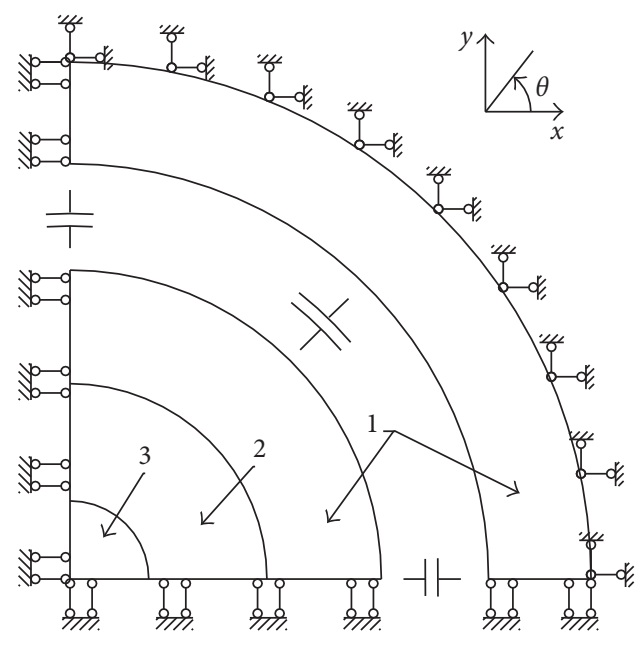

(b)

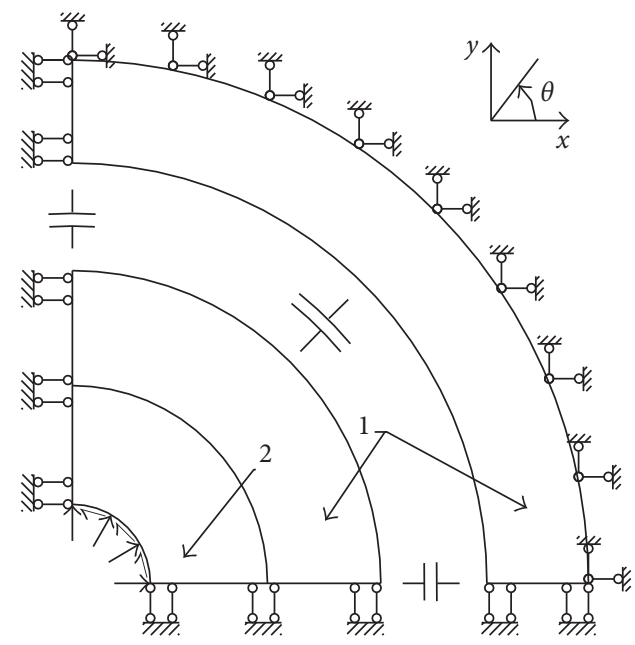

(c)

FIgURE 4: Schematic diagram of finite-element model: (a) before freezing; (b) after freezing and before excavation; (c) after excavation. 1, unfrozen rock; 2 , frozen wall; 3 , rock to be excavated.

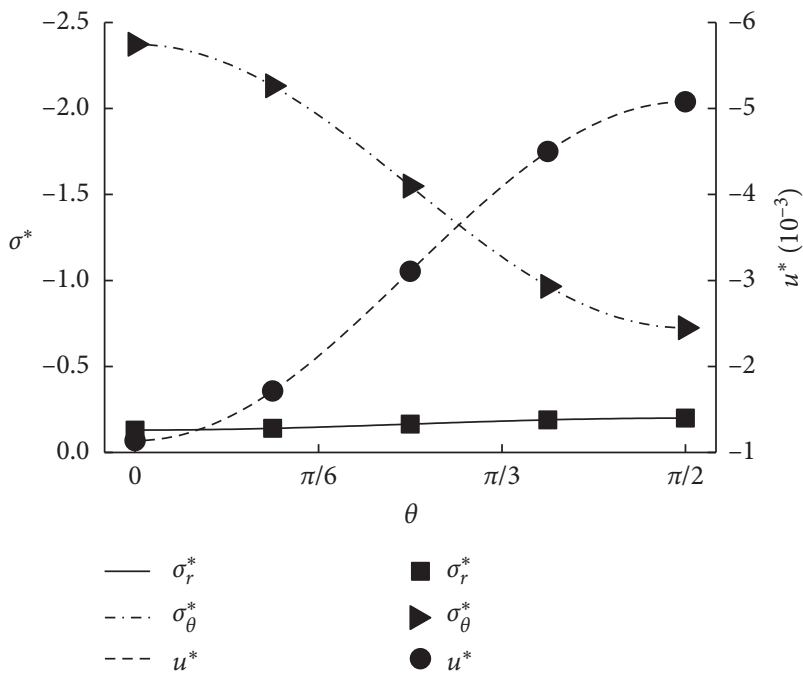

(a)
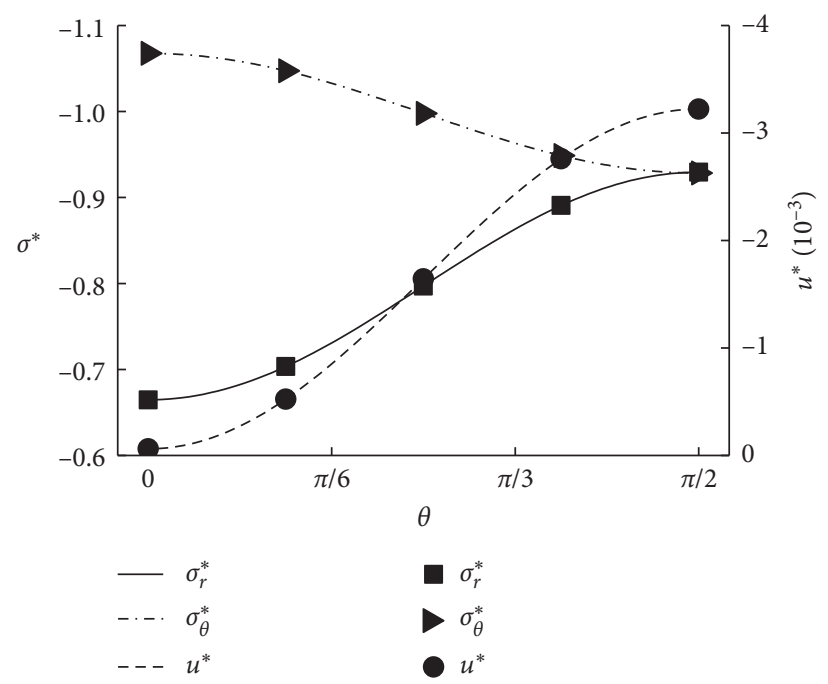

(b)

FiguRE 5: Variation of $\sigma_{r}^{*}, \sigma_{\theta}^{*}$, and $u^{*}$ along the (a) inner and (b) outer edges of the frozen wall. 


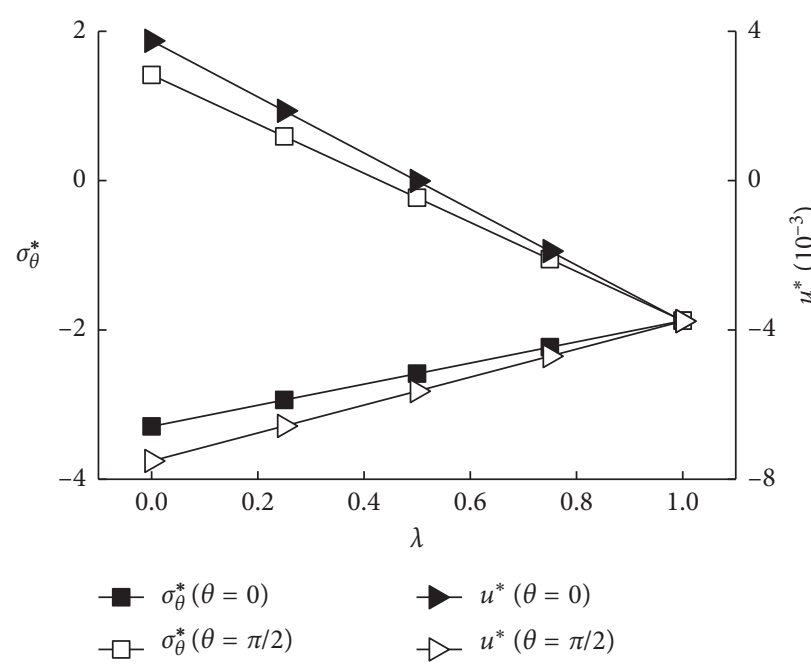

(a)

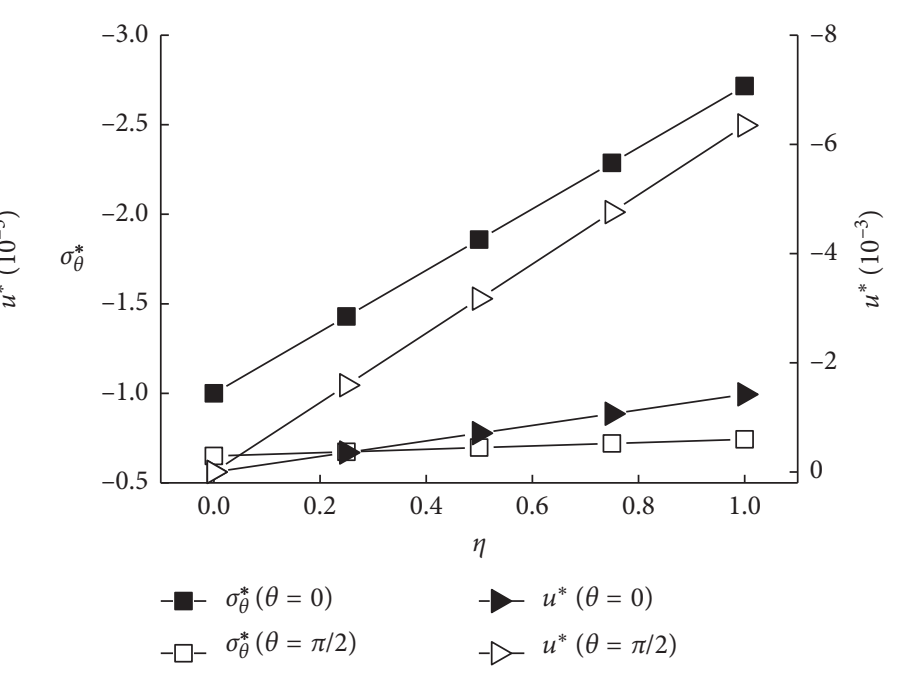

(b)

FIgURE 6: $\sigma_{\theta}^{*}$ and $u^{*}$ at the inner edge of the inhomogeneous frozen wall as a function of (a) $\lambda$ and (b) $\eta$.

TABle 2: $\phi_{1}, \phi_{2}$, and $\phi_{3}$ at the outer edge of the frozen wall and for different freezing modes.

\begin{tabular}{|c|c|c|c|c|c|c|}
\hline \multirow{3}{*}{ Freezing modes } & \multicolumn{6}{|c|}{ Coefficient of the unloading ratio } \\
\hline & \multicolumn{2}{|c|}{$\phi_{1}$} & \multicolumn{2}{|c|}{$\phi_{2}$} & \multicolumn{2}{|c|}{$\phi_{3}$} \\
\hline & IF model & HF model & IF model & HF model & IF model & HF model \\
\hline Single-circle freezing & 0.0426 & 0.0438 & 0.306 & 0.320 & -0.167 & -0.161 \\
\hline Double-circle freezing & 0.0226 & 0.0227 & 0.153 & 0.150 & -0.0876 & -0.0828 \\
\hline Triple-circle freezing & 0.0122 & 0.0119 & 0.0744 & 0.0737 & -0.0424 & -0.0404 \\
\hline
\end{tabular}

differ slightly between the two models (Table 2). Figure 7 shows the parameters $\phi_{1}, \phi_{2}$, and $\phi_{3}$ as functions of the contact-surface number $j$ for single-circle soil freezing. The stress state in the frozen wall is complicated; the parameters related with nonuniform load $\left(\phi_{2}\right.$ and $\left.\phi_{3}\right)$ vary nonmonotonically, unlike $\phi_{1}$ which decreases monotonically along the radius. $\phi_{2}$ initially increases and then decreases along the radius, whereas $\phi_{3}$ decreases sharply and then rises gradually. In addition, calculations indicate that $\phi_{1}, \phi_{2}$, and $\phi_{3}$ gradually approach zero with increasing radius when the unfrozen stratum is inhomogeneous.

From Figure 7, we see that $\phi_{1}$ and $\phi_{2}$ are always positive along the radius, but $\phi_{3}$ is negative for $j>5$. This indicates that the excavation should decrease the radial force exerted by the frozen wall on the surrounding rock and increase the tangential pressure. To explain this phenomenon, we extract the orientations of greatest principal stress in the frozen wall before and after excavation. The initial direction of maximal principal stress is $\theta=\pi / 2$ ( $y$ direction); after excavation, the direction deflects by varying degrees (Figure 8 ), which is a sign of stress redistribution. Figure 8 shows the deviation angles of principal stress at different locations. The greatest deflection occurs at the intersection of the inner edge of the frozen wall and in the direction $\theta=\pi / 2$, where the orientation changes $90^{\circ}$ from the $y$ direction to the $x$ direction. The redistribution of stress may be the reason for the increase in the tangential interaction force between the frozen wall and surrounding rock.

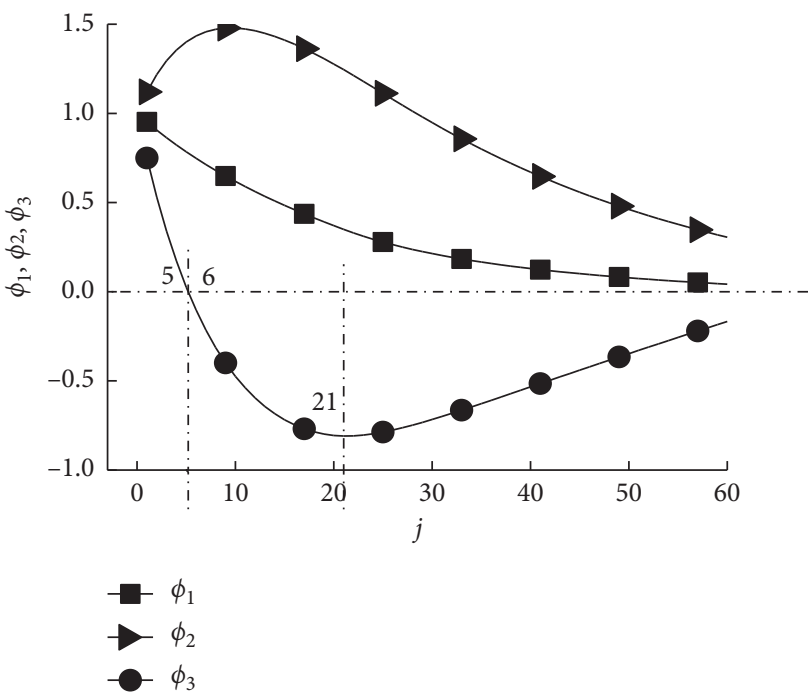

Figure 7: $\phi_{1}, \phi_{2}$, and $\phi_{3}$ on each contact face as a function of contact-surface number $j$ for single-circle soil freezing.

Curve 1 shows the initial directions of the maximal principal stress at different locations. Curves 2-5 represent a quarter of a ring of radius of 4.2, 6.3, 8.2, and 10.2 meters, respectively, and show the deflection angles of the principal stress in the circumferential direction. 


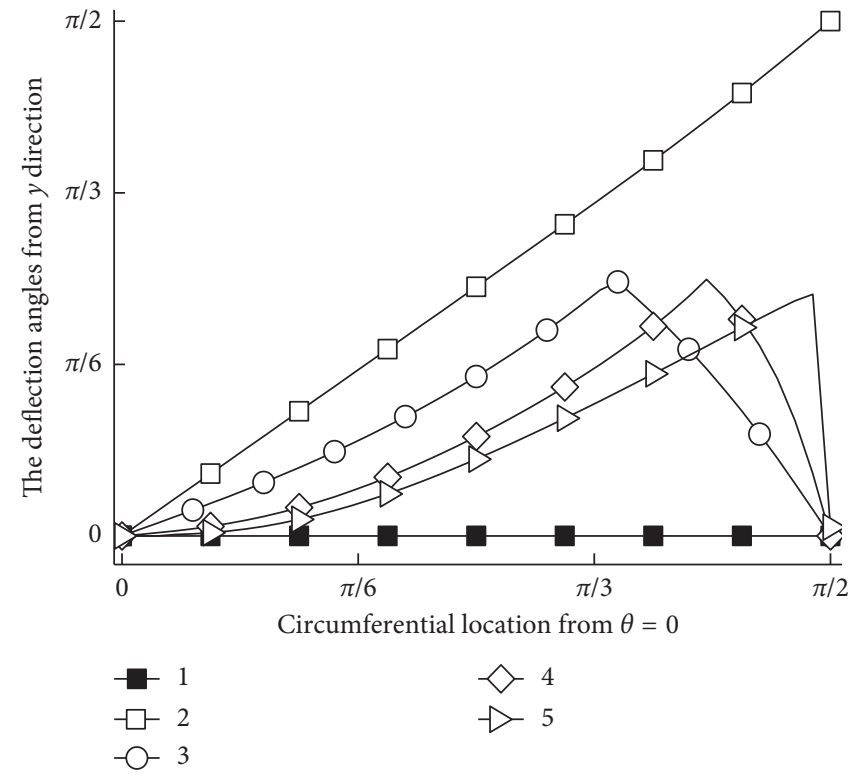

FiguRE 8: Deflection angles of maximal principal stress at different locations.

Table 2 shows $\phi_{1}, \phi_{2}$, and $\phi_{3}$ for different thicknesses of the frozen wall and for the two calculation models. With increasing thickness, the absolute values of all three coefficients decrease. The excavation unloading has less of an effect on the interaction between the frozen wall and surrounding rock, which means that the stress at the outer edge of the frozen wall approaches the initial ground stress.

To summarize, considering the heterogeneity of the frozen wall is helpful to understand the complex state of the interior stress. After excavation, the directions of principal stress change because of the nonuniform crustal stress field and the tangential interaction force between the frozen wall and surrounding rock increases. These factors should be considered when designing frozen walls.

\subsection{Comparison of Radial Distributions of Circumferential} Stress and Radial Displacement in Both Mechanical Models. The radial distributions of circumferential stress for both the mechanical models are shown in Figure 9. $\widetilde{\sigma}_{\theta}^{*}$ and $\bar{\sigma}_{\theta}^{*}$ represent the circumferential stress for the IF model and HF model, respectively. The decrease in $\widetilde{\sigma}_{\theta}^{*}$, which differs from $\bar{\sigma}_{\theta}^{*}$, has inflection points near the freezing pipes. Combined with Figure 3, we find that the discrepancy between $\widetilde{\sigma}_{\theta}^{*}$ and $\bar{\sigma}_{\theta}^{*}$ is closely related to the temperature distribution in the two models. For below-average temperature, $\left|\widetilde{\sigma}_{\theta}^{*}\right|<\left|\bar{\sigma}_{\theta}^{*}\right|$, whereas with above-average temperature, $\left|\widetilde{\sigma}_{\theta}^{*}\right|>\left|\bar{\sigma}_{\theta}^{*}\right|$. This explains why $\left|\widetilde{\sigma}_{\theta}^{*}\right|<\left|\bar{\sigma}_{\theta}^{*}\right|$ at the inner and outer boundaries of the frozen wall and $\left|\widetilde{\sigma}_{\theta}^{*}\right|>\left|\bar{\sigma}_{\theta}^{*}\right|$ near freezing pipes. Furthermore, upon increasing the thickness of the frozen wall, the temperature in the HF model approaches the minimum temperature of the IF model, and the gap between $\widetilde{\sigma}_{\theta}^{*}$ and $\widetilde{\sigma}_{\theta}^{*}$ decreases outside the innermost freezing pipes as the lowertemperature region of the IF model widens. This analysis illustrates that the homogeneous model cannot accommodate the frozen-wall design because of the significant discrepancy between the distributions of $\widetilde{\sigma}_{\theta}^{*}$ and $\bar{\sigma}_{\theta}^{*}$.
Copious calculations indicate that the radial displacement in all cases and models decreases nonlinearly in the radial direction, with only minor differences occurring in magnitude. Therefore, we show them in Figure 10 as functions of radius only for single-circle freezing.

4.6. Comparison between Models of Stress and Displacement at Inner Edge of Frozen Wall. Because the mechanical state of the inner edge is the key factor in the design of frozen walls, we calculate and sum the stress and the displacement of the inner edge for different freezing modes (Table 3). The elastic modulus depends on the temperature. The results of the comparison indicate that, at the inner edge, the absolute value of the circumferential stress of the IF model is less than that of the HF model in any freezing mode, and the discrepancy increases with thickness: in going from singlecircle freezing to triple-circle freezing, the discrepancy increases from $12.41 \%$ to $18.03 \%$ at $\theta=\pi / 2$. The reason behind the variation in discrepancy between the two circumferential stresses may be the increasing temperature difference at the inner edge. In this example calculation, the temperature of the inner margin for the IF model is independent of the thickness of the frozen wall, whereas the average temperature for the HF model decreases with thickness. Thus, the discrepancy in the temperature of the inner margin between the two models correlates positively with frozen-wall thickness.

Table 3 also shows the results for calculating the displacements under different conditions. For small frozen-wall thickness, the inner edge for the IF model is less displaced than for the HF model. In addition, the deviation decreases with increasing thickness. Finally, for the IF model, the displacement increases for triple-circle freezing.

For the IF model and frozen rock, the absolute value of the displacement of the inner edge decreases with increasing frozen-wall thickness. However, the relationship is different 


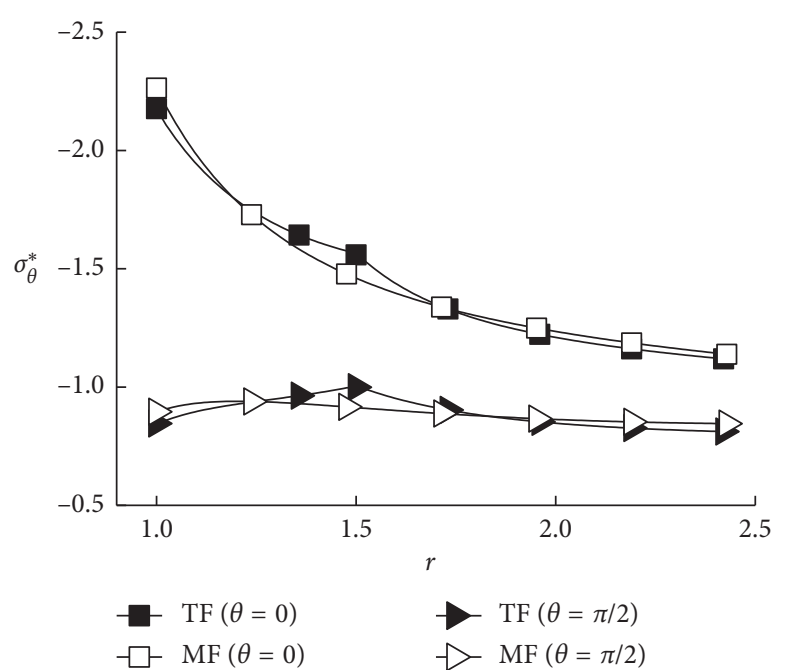

(a)

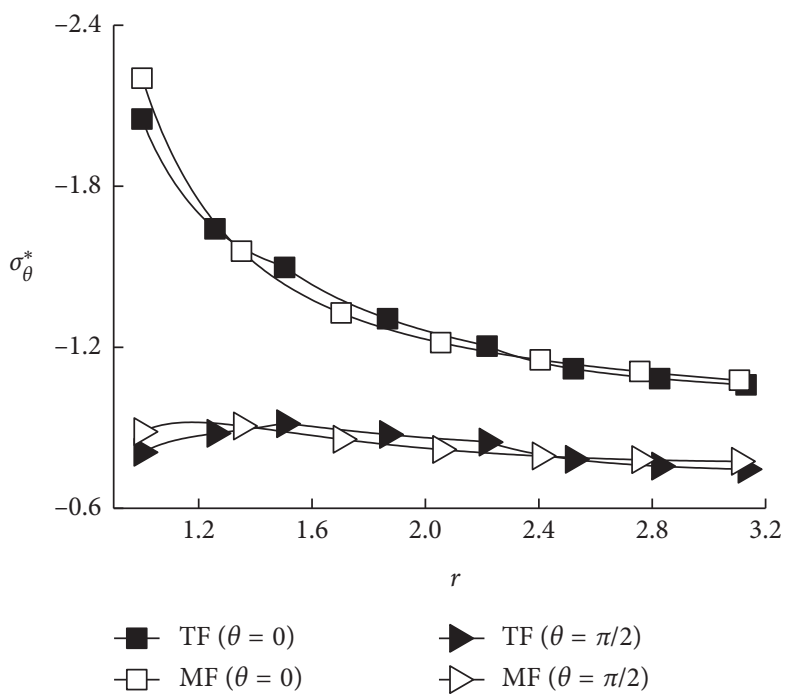

(c)

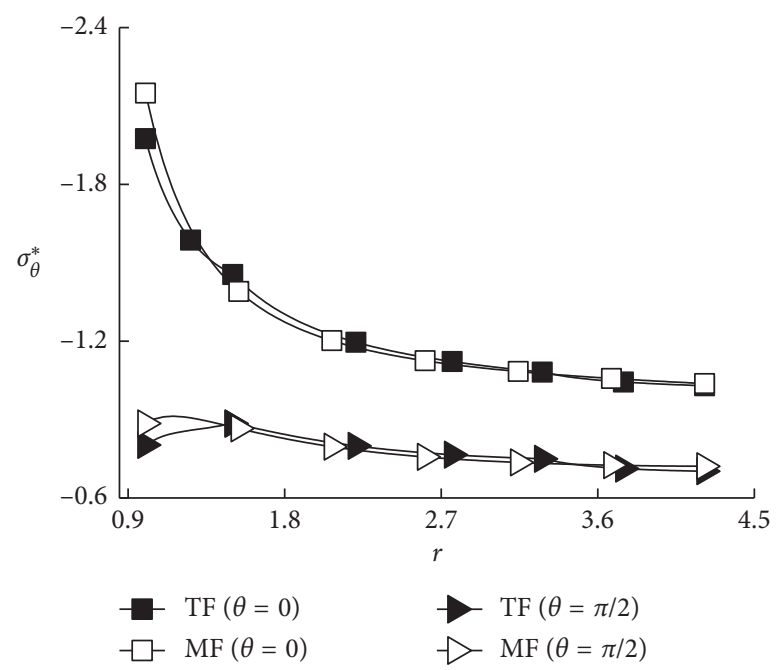

(e)

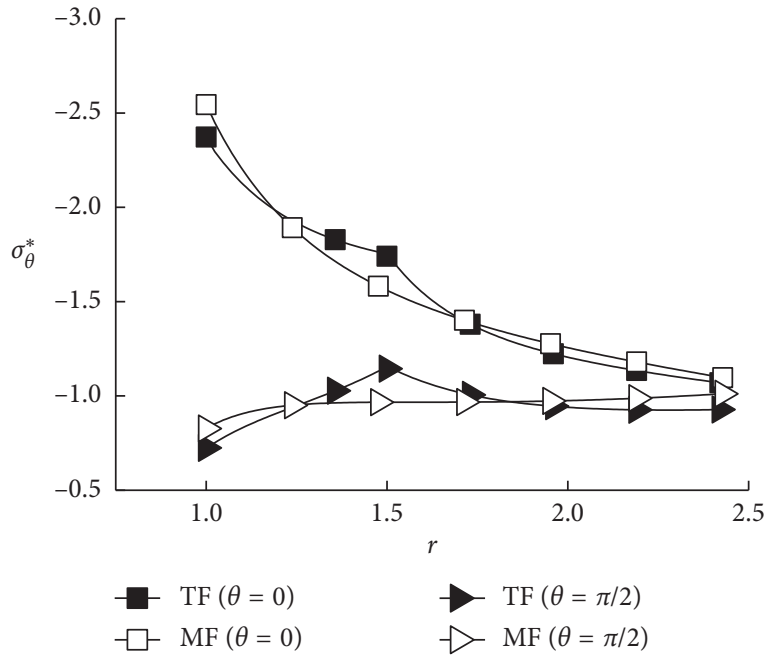

(b)

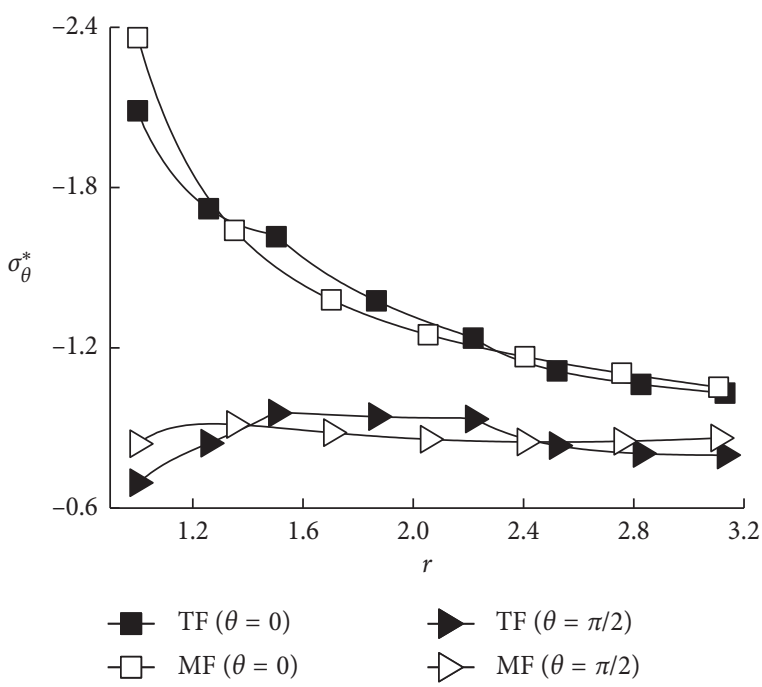

(d)

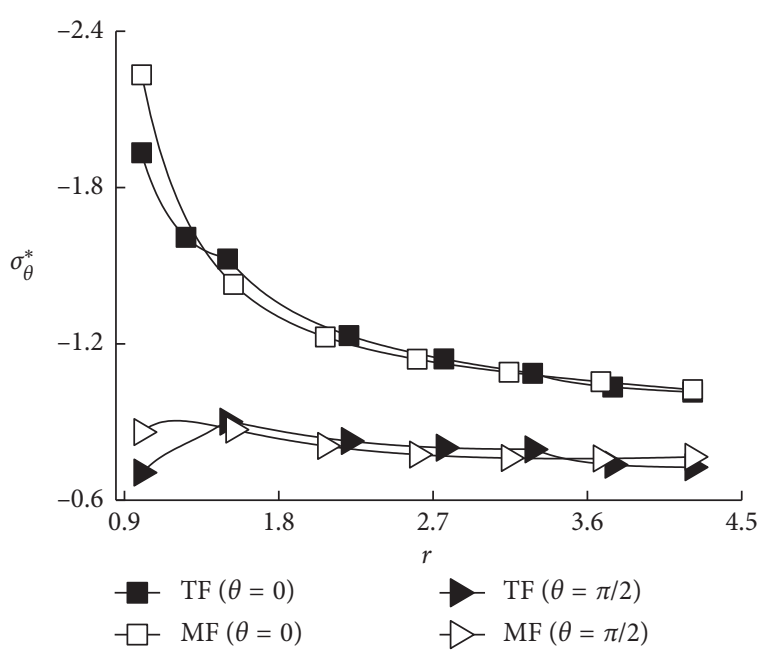

(f)

FIGURe 9: Distribution graphs of $\sigma_{\theta}^{*}-r$ : (a) single-circle pipes and rock freezing; (b) single-circle pipes and soil freezing; (c) double-circle pipes and rock freezing; (d) double-circle pipes and soil freezing; (e) triple-circle pipes and rock freezing; (f) triple-circle pipes and soil freezing. 


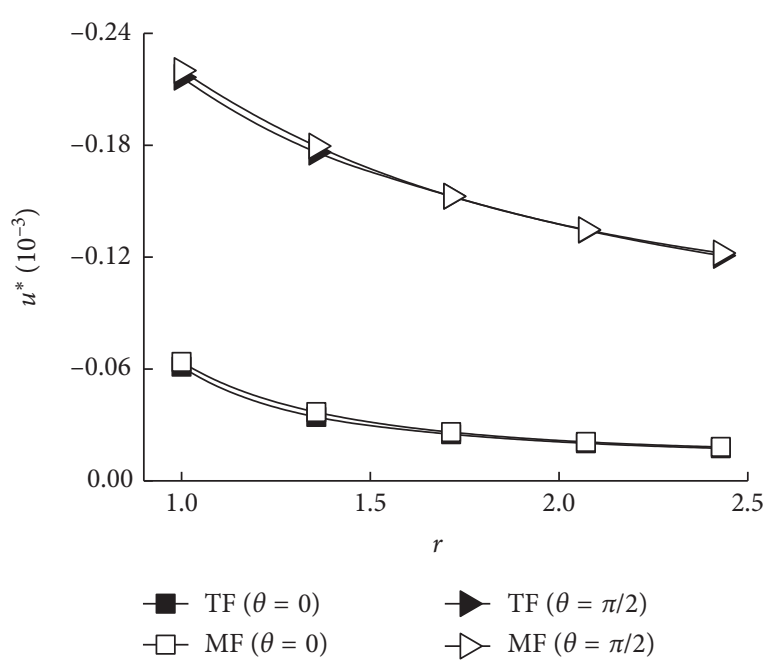

(a)

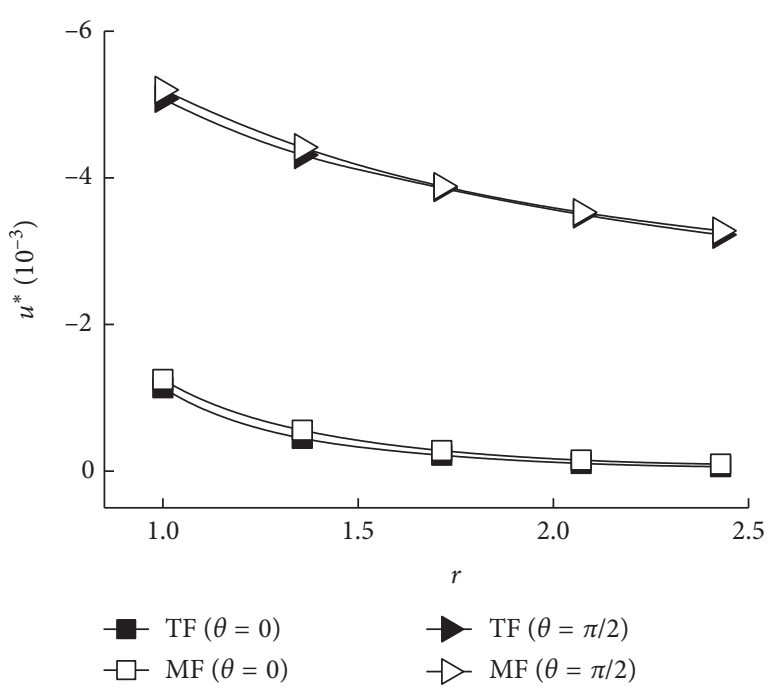

(b)

FIgURE 10: Graphs of $u^{*}$ versus $r$ for single-circle freezing: (a) frozen rock; (b) frozen soil.

TABLE 3: Circumferential stress $\sigma_{\theta}^{*}$ and radial displacement $u^{*}$ of the inner edge for the IF model and HF model under different freezing modes.

\begin{tabular}{|c|c|c|c|c|c|c|c|c|}
\hline \multirow[t]{2}{*}{ Freezing modes } & \multirow[t]{2}{*}{ Formation conditions } & \multirow[t]{2}{*}{ Locations } & \multicolumn{2}{|c|}{$\sigma_{\theta}^{*}$} & \multicolumn{2}{|c|}{$u^{*}$} & \multicolumn{2}{|c|}{$\begin{array}{c}\text { Reduction rates } \\
(\%)\end{array}$} \\
\hline & & & IF model & HF model & IF model & HF model & $\sigma_{\theta}^{*}$ & $u^{*}$ \\
\hline \multirow{4}{*}{ Single-circle pipes } & \multirow{2}{*}{1} & 0 & -2.176 & -2.263 & -0.0612 & -0.0638 & -3.84 & -4.04 \\
\hline & & $\pi / 2$ & -0.846 & -0.900 & -0.216 & -0.220 & -5.57 & -1.66 \\
\hline & \multirow{2}{*}{2} & 0 & -2.372 & -2.544 & -1.137 & -1.250 & -6.74 & -9.05 \\
\hline & & $\pi / 2$ & -0.724 & -0.827 & -5.077 & -5.200 & -12.41 & -2.37 \\
\hline \multirow{4}{*}{ Double-circle pipes } & \multirow{2}{*}{1} & 0 & -2.051 & -2.202 & -0.0585 & -0.0582 & -6.89 & 0.40 \\
\hline & & $\pi / 2$ & -0.808 & -0.885 & -0.192 & -0.193 & -8.70 & -0.526 \\
\hline & \multirow{2}{*}{2} & 0 & -2.087 & -2.361 & -1.209 & -1.207 & -11.59 & -0.163 \\
\hline & & $\pi / 2$ & -0.695 & -0.841 & -4.008 & -4.030 & -17.36 & -0.549 \\
\hline \multirow{4}{*}{ Triple-circle pipes } & \multirow{2}{*}{1} & 0 & -1.975 & -2.149 & -0.059 & -0.055 & -8.12 & 6.86 \\
\hline & & $\pi / 2$ & -0.803 & -0.885 & -0.178 & -0.176 & -9.32 & 1.41 \\
\hline & \multirow{2}{*}{2} & 0 & -1.933 & -2.233 & -1.281 & -1.161 & -13.41 & 10.35 \\
\hline & & $\pi / 2$ & -0.706 & -0.861 & -3.48 & -3.41 & -18.03 & 2.20 \\
\hline
\end{tabular}

PS: reduction rate $=($ IF model $-\mathrm{HF}$ model $) / \mathrm{HF}$ model $\times 100 \%$.

in frozen soil: at $\theta=0$, the absolute value of the displacement increases with thickness, whereas the opposite is true at $\theta=\pi / 2$. These calculation results coincide with actual physical phenomena. Frozen rock is relatively strong and can strongly interact with unfrozen ground, which could restrict the deformation of the frozen wall. However, for frozen soil, the lower strength and weaker interaction between frozen and unfrozen strata should weaken the constraints for frozen soil.

The circumferential stress of the inner edge is a primary concern in the frozen-wall design. Based on the analysis above, we see that the circumferential stress is smaller for the IF model. Consequently, the HF model cannot completely describe the bearing property and the deformation performance of the frozen wall. This will result in a significant waste of refrigeration power, particularly for triplecircle freezing.

\section{Conclusions}

This study presents a plane-strain model that accounts for the inhomogeneity of a frozen wall and excavation unloading under a nonuniform ground stress. The model is based on the superposition of thin concentric cylinders with two types of contact conditions: complete contact and smooth contact. The analytical solution is derived and verified by comparing it with the result of a finite-element simulation. The proposed model is applicable for any case with radial variation of the 
parameters and accurately reflects the characteristics of a frozen-soil wall, the surrounding earth mass, and the stress state.

For the nonuniform ground stress field, unlike the uniform geostress, the principal stress rotates after excavation, which increases the tangential interaction force between the frozen wall and surrounding rock.

For equal-ratio excavation unloading (i.e., the radial unloading equals the tangential unloading at the inner edge of the frozen wall), the radial response differs from that of the tangential stress at the outer edge of the frozen wall. The unloading proportion of the uniform term and nonuniform term of radial force and the unloading rate of tangential pressure are $\phi_{1} \eta, \phi_{2} \eta$, and $\phi_{3} \eta$, respectively, and the parameters $\phi_{2}$ and $\phi_{3}$ vary nonmonotonically, whereas $\phi_{1}$ decreases monotonically with the radius.

The tangential stress $\sigma_{\theta}^{*}$ and the radial displacement $u^{*}$ at the inner edge of the frozen wall correlate linearly with the nonuniform coefficient of ground press $\lambda$ and the excavation unloading ratio $\eta$. The inner edge of the frozen wall may incur tensile stress and large inward pulling when $\lambda$ is relatively small $(\lambda \leq 0.43)$. Consequently, measurements should be made to ensure the safety of the shaft.

The difference in the results of the two models is mainly embodied in circumferential stress. $\left|\widetilde{\sigma}_{\theta}^{*}\right|<\left|\bar{\sigma}_{\theta}^{*}\right|\left(\left|\widetilde{\sigma}_{\theta}^{*}\right|>\left|\bar{\sigma}_{\theta}^{*}\right|\right)$, when the temperature of the IF model is below (above) average.

When the frozen wall is relatively thick, the inner-edge circumferential stress of the frozen wall for the IF model is less than that for the HF model; the decrease is $8.12 \% \sim 9.32 \%$ for frozen rock and $13.41 \% \sim 18.03 \%$ for frozen soil (Table 3). Therefore, it is economical and reasonable to use the IF model to design frozen walls.

\section{Data Availability}

The data used to support the findings of this study are included within the article.

\section{Conflicts of Interest}

The authors declare that there are no conflicts of interest regarding the publication of this paper.

\section{Acknowledgments}

The work presented in this paper was supported by the National Natural Science Foundation of China (Grant no. 41472224) and the National Key Research and Development Program of China (Grant no. 2016YFC0600904).

\section{References}

[1] O. B. Andersland and B. Ladanyi, Frozen Ground Engineering, John Wiley \& Sons Inc., Hoboken, NJ, USA, 2003.

[2] J. X. Ren, J. L. Sun, K. Zhang et al., "Mechanical properties and temperature field of inclined frozen wall in water-rich sand stratum," Rock and Soil Mechanics, vol. 38, no. 5, pp. 14051412, 2017.
[3] T. J. Zhou and G. Q. Zhou, "Calculation method of frozen soil wall thickness for inclined shaft in consideration of the shaft inclination," Journal of China University of Mining and Technology, vol. 45, no. 3, pp. 514-520, 2016.

[4] H. Song, H. Cai, Z. Yao, C. Rong, and X. Wang, "Finite element analysis on 3D freezing temperature field in metro cross passage construction," Procedia Engineering, vol. 165, pp. 528-539, 2016.

[5] Y. Kang, Q. Liu, Y. Cheng, and X. Liu, "Combined freezesealing and new tubular roof construction methods for seaside urban tunnel in soft ground," Tunnelling and Underground Space Technology, vol. 58, pp. 1-10, 2016.

[6] S. Li, Y. Lai, M. Zhang, and S. Zhang, "Minimum ground prefreezing time before excavation of Guangzhou subway tunnel," Cold Regions Science and Technology, vol. 46, no. 3, pp. 181-191, 2006.

[7] J. H. Huang, "Simulation analysis and experimental test on frost deformation of freezing waterproof curtain in deep foundation pit," Chinese Journal of Rock Mechanics and Engineering, vol. 28, no. 12, pp. 2554-2560, 2009.

[8] X. D. Hu, "A mechanical model of interaction of frozen soil wall and surrounding earth mass in unloaded state," Journal of China Coal Society, vol. 26, no. 5, pp. 507-511, 2001.

[9] W. H. Yang, Z. J. Yang, T. Han et al., "Elastic design theory of frozen soil wall based on interaction between frozen soil wall and surrounding rock," Chinese Journal of Geotechnical Engineering, vol. 34, no. 3, pp. 516-519, 2012.

[10] B. Liu, C. J. Song, T. Li et al., "Interaction mechanism of deepburied frozen soil wall and surrounding earth mass in excavation unloaded state," Journal of China Coal Society, vol. 37, no. 11, pp. 1834-1840, 2012.

[11] W. B. Yuan, Y. M. Ma, and K. D. Chen, "Analysis of stresses of the nonhomogeneous elastic frozen wall," Journal of China Coal Society, vol. 2, pp. 44-50, 1983.

[12] C. A. You, "Analysis of elasto-plastic stresses in inhomogeneous ice-wall and calculation of ice-wall," Journal of China Coal Society, vol. 2, pp. 80-88, 1986.

[13] G. R. Zhong, G. Q. Zhou, J. Z. Wang, and X. Y. Shang, "Viscoelastic analysis of heterogeneous frozen wall in deep alluvium," Journal of China Coal Society, vol. 35, no. 3, pp. 397-401, 2010.

[14] X. D. Hu, C. Shu, and S. Y. She, "Elastic-plastic analytical solution for functionally graded material frozen soil wall with parabolic property under uniform load," Journal of China Coal Society, vol. 37, no. 3, pp. 379-384, 2012.

[15] X. Y. Cao, J. H. Zhao, and C. G. Zhang, "Elastoplastic stress analysis of frozen soil wall based on unified strength theory," Rock and Soil Mechanics, vol. 38, no. 3, pp. 769-809, 2017.

[16] S. C. Li and M. B. Wang, "Elastic analysis of stressdisplacement field for a lined circular tunnel at great depth due to ground loads and internal pressure," International Journal of Rock Mechanics and Mining Sciences, vol. 45, no. 4, pp. 486-494, 2008.

[17] A. Z. Lu, N. Zhang, Y. Q. Xu, and P. Cui, "Stress-displacement solution for a lined vertical borehole due to non-axisymmetric in situ stresses," International Journal of Rock Mechanics and Mining Sciences, vol. 57, pp. 64-74, 2013.

[18] C. Carranza-Torres, B. Rysdahl, and M. Kasim, "On the elastic analysis of a circular lined tunnel considering the delayed installation of the support," International Journal of Rock Mechanics and Mining Sciences, vol. 61, pp. 57-85, 2013.

[19] T. D. Y. F. Simanjuntak, M. Marence, A. E. Mynett, and A. J. Schleiss, "Pressure tunnels in non-uniform in situ stress 
conditions," Tunnelling and Underground Space Technology, vol. 42, pp. 227-236, 2014.

[20] R. S. Yang and Q. X. Wang, "Elastic analysis and design of circular horizontal frozen wall based on interaction between frozen wall and surrounding rock," Journal of China Coal Society, vol. 41, no. 5, pp. 1069-1077, 2016.

[21] Y. Wang and W. H. Yang, "Elastic design theory of circular horizontal frozen wall," Journal of China Coal Society, vol. 40, no. 9, pp. 2049-2056, 2015.

[22] Y. Wang and W. H. Yang, "Elastic stress analysis on circular frozen wall in bi-directional unequal ground stress field under unloading condition," Journal of Mining and Safety Engineering, vol. 33, no. 3, pp. 486-493, 2016.

[23] C. Zhang, Study on Key Construction Technologies of the New Monolayer Freezing Shaft Lining in Deep Water-Rich Bed Rock, China University of Mining and Technology, Xuzhou, Jiangsu, China, 2012, in Chinese.

[24] C. Atkinson and D. A. Eftaxiopoulos, "Numerical and analytical solutions for the problem of hydraulic fracturing from a cased and cemented wellbore," International Journal of Solids and Structures, vol. 39, no. 6, pp. 1621-1650, 2002.

[25] I. D. Moore and J. R. Booker, "Behaviour of buried flexible cylinder under the influence of nonuniform hoop compression," International Journal of Solids and Structures, vol. 21, no. 9, pp. 943-956, 1985.

[26] J. P. Carter and J. R. Booker, "Elastic consolidation around a lined circular tunnel," International Journal of Solids and Structures, vol. 20, no. 6, pp. 589-608, 1984.

[27] I. D. Moore and J. R. Booker, "Simplified theory for the behaviour of buried flexible cylinders under the influence of uniform hoop compression," International Journal of Solids and Structures, vol. 21, no. 9, pp. 929-941, 1985. 


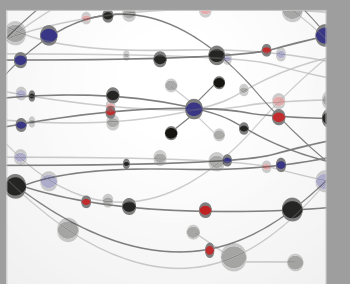

The Scientific World Journal
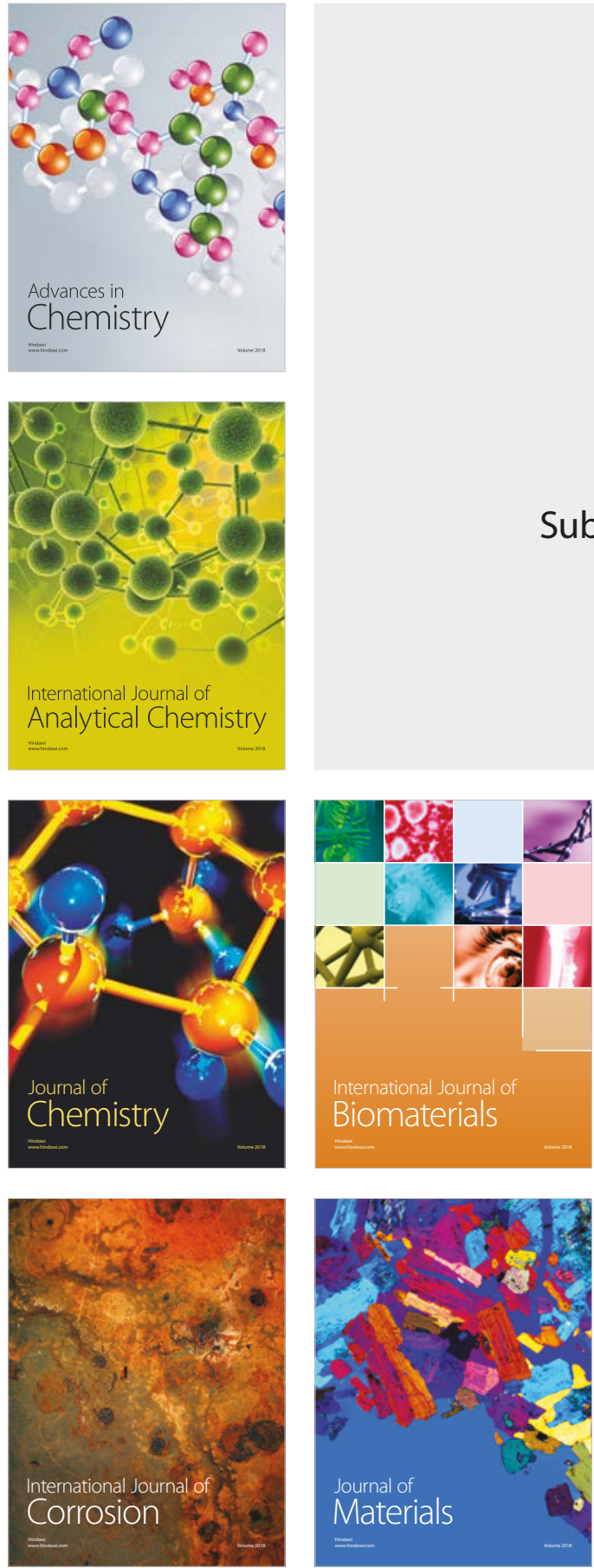

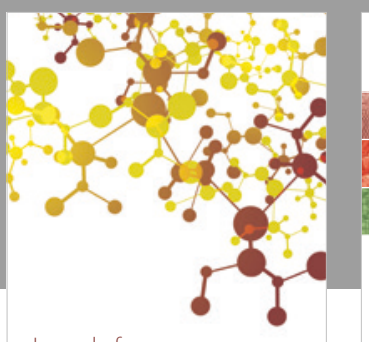

Journal of

Applied Chemistry
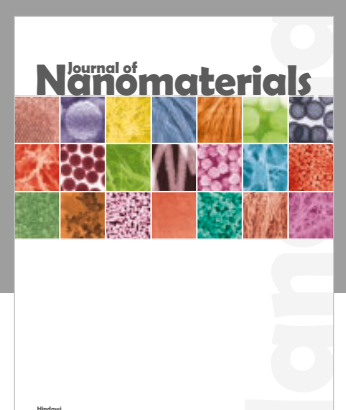

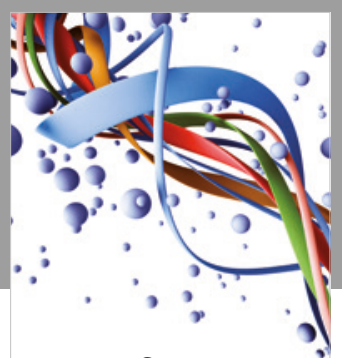

Scientifica

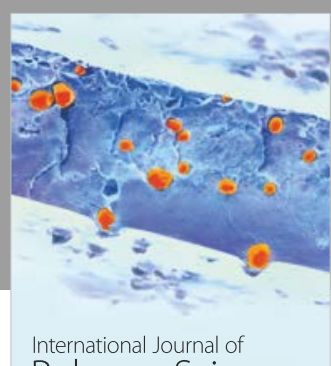

Polymer Science

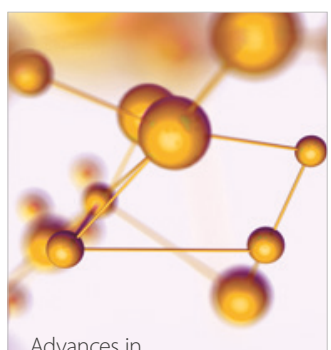

Physical Chemistry
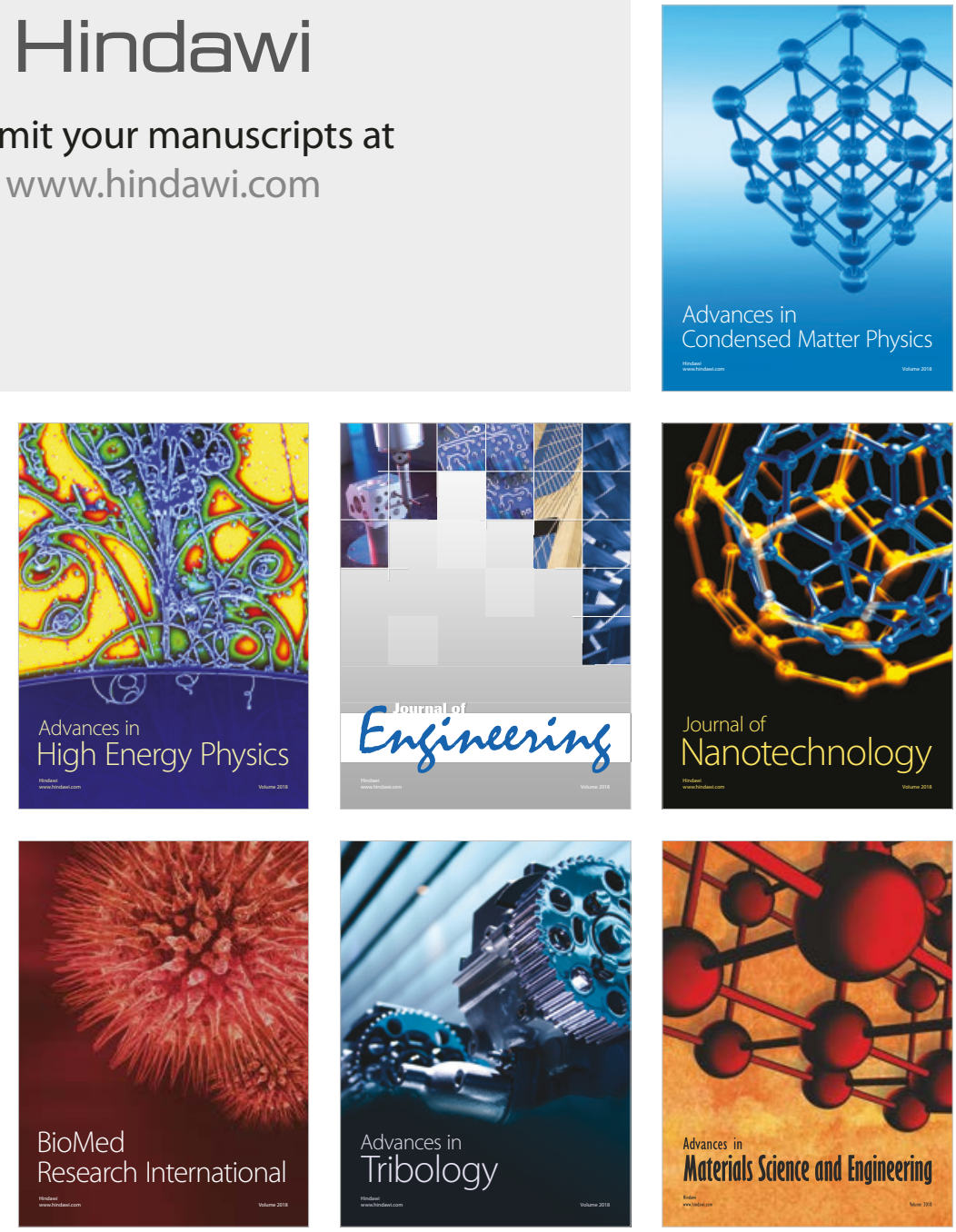